\title{
KiDS+VIKING-450 and DES-Y1 combined: Cosmology with cosmic shear
}

\author{
S. Joudaki ${ }^{1}$, H. Hildebrandt ${ }^{2,3}$, D. Traykova ${ }^{1}$, N. E. Chisari ${ }^{1}$, C. Heymans ${ }^{4,2}$, A. Kannawadi ${ }^{5}$, K. Kuijken ${ }^{5}$,
} A. H. Wright ${ }^{2,3}$, M. Asgari ${ }^{4}$, T. Erben ${ }^{3}$, H. Hoekstra ${ }^{5}$, B. Joachimi ${ }^{6}$, L. Miller ${ }^{1}$, T. Tröster ${ }^{4}$, and J. L. van den Busch ${ }^{2,3}$

1 Department of Physics, University of Oxford, Denys Wilkinson Building, Keble Road, Oxford OX1 3RH, UK e-mail: shahab.joudaki@physics.ox.ac.uk

2 Ruhr-Universität Bochum, Astronomisches Institut, German Centre for Cosmological Lensing, Universitätsstr. 150, 44801 Bochum, Germany

3 Argelander-Institut für Astronomie, Universität Bonn, Auf dem Hügel 71, 53121 Bonn, Germany

${ }^{4}$ Institute for Astronomy, University of Edinburgh, Royal Observatory, Blackford Hill, Edinburgh EH9 3HJ, UK

5 Leiden Observatory, Leiden University, PO Box 9513, 2300 RA Leiden, The Netherlands

6 Department of Physics and Astronomy, University College London, Gower Street, London WC1E 6BT, UK

Received 22 June 2019/ Accepted 22 April 2020

\begin{abstract}
We present a combined tomographic weak gravitational lensing analysis of the Kilo Degree Survey (KV450) and the Dark Energy Survey (DES-Y1). We homogenize the analysis of these two public cosmic shear datasets by adopting consistent priors and modeling of nonlinear scales, and determine new redshift distributions for DES-Y1 based on deep public spectroscopic surveys. Adopting these revised redshifts results in a $0.8 \sigma$ reduction in the DES-inferred value for $S_{8}$, which decreases to a $0.5 \sigma$ reduction when including a systematic redshift calibration error model from mock DES data based on the MICE2 simulation. The combined KV450+DES-Y1 constraint on $S_{8}=0.762_{-0.024}^{+0.025}$ is in tension with the Planck 2018 constraint from the cosmic microwave background at the level of $2.5 \sigma$. This result highlights the importance of developing methods to provide accurate redshift calibration for current and future weak-lensing surveys.
\end{abstract}

Key words. cosmology: observations - galaxies: photometry - gravitational lensing: weak - surveys

\section{Introduction}

Weak gravitational lensing tomography has entered the phase of precision cosmology, with observational constraints on the best-measured parameter, $S_{8}=\sigma_{8} \sqrt{\Omega_{\mathrm{m}} / 0.3}$, at a level of precision $\lesssim 5 \%$ for all current surveys (Hildebrandt et al. 2020, hereafter H20; Troxel et al. 2018, hereafter T18; Hikage et al. 2019; Joudaki et al. 2017; Jee et al. 2016). Here, $\sigma_{8}$ refers to the rootmean-square of the linear matter overdensity field on $8 \mathrm{~h}^{-1} \mathrm{Mpc}$ scales, and $\Omega_{\mathrm{m}}$ is the present mean density of nonrelativistic matter relative to the critical density. This phase has been reached as a result of the success in accounting for the systematic uncertainties that affect the measurements. However, as the statistical precision of weak-lensing surveys increases with depth and area, the requirements on their ability to control systematic uncertainties increase as well. In Hildebrandt et al. (2017), it was shown that the contribution of systematic uncertainties to the total error budget for the Kilo Degree Survey (KiDS; Kuijken et al. 2015) is comparable to that of the statistical uncertainties. Given the similar constraining power of concurrent weak-lensing surveys, such as the Dark Energy Survey (DES; Abbott et al. 2018a) and the Subaru Hyper Suprime-Cam survey (HSC; Aihara et al. 2018), a continued reduction in the systematic uncertainties is crucial to obtain unbiased cosmological constraints and to exploit the full statistical power of current and future weak-lensing datasets.
The most notable systematic uncertainties pertain to the intrinsic alignment (IA) of galaxies, additive and multiplicative shear calibration, baryonic feedback affecting the nonlinear matter power spectrum, and photometric redshift errors (see Mandelbaum 2018 and references therein). All current weak-lensing surveys have reached a statistical precision where notable changes to the cosmological parameter constraints are found when accounting for these systematic uncertainties in the analysis (e.g. Hikage et al. 2019; T18; H20). The expectation is that the final parameter constraints are robust when marginalized over all known systematics. This is generally well motivated through the vast range of checks and extensions of the systematic models beyond the standard approach considered by these surveys. The uncertainty in the redshift distributions, $n(z)$, of weakly lensed galaxies is, however, more difficult to account for, and has been shown to be the only systematic uncertainty to impact the posterior mean of $S_{8}$ by $\sim 1 \sigma(\mathrm{H} 20)$.

The redshift uncertainty is arguably the most challenging systematic to control in both current and future lensing surveys. In KiDS, the estimation of the redshift distributions has benefited from the fully overlapping near-infrared imaging data from the VISTA Kilo-Degree Infrared Galaxy Survey (VIKING; Edge et al. 2013). The combined KiDS and VIKING dataset ('KiDS+VIKING-450' or 'KV450'; Wright et al. 2019) has allowed for an increased precision in the estimation of photometric redshifts that are used to assign sources to tomographic 
bins. In addition, KiDS targets deep pencil-beam spectroscopic surveys permitting the redshift distributions to be determined via the weighted direct estimation, or 'DIR', approach (Lima et al. 2008; Hildebrandt et al. 2017; H20), which is fully decoupled from the photo- $z$. This DIR method assigns KiDS sources to spectroscopic galaxies by a $k$-nearest-neighbor matching in order to estimate weights for the spectroscopic objects. The weighted distribution of spectroscopic redshifts can then be used to estimate the $n(z)$ of the sources. The uncertainty $\Delta z_{i}$ in the mean redshift of each tomographic bin $i$ is obtained from a spatial bootstrap resampling of the spectroscopic calibration sample and propagated in the cosmological analysis as $n_{i}(z) \rightarrow$ $n_{i}\left(z-\Delta z_{i}\right)(\mathrm{H} 20)$.

The DIR approach has been found to produce cosmological results consistent with other $n(z)$ estimation techniques, such as the angular cross-correlation of photometric and spectroscopic galaxy samples (where the spectroscopic samples are obtained from overlapping wide and shallow surveys; Morrison et al. 2017; Johnson et al. 2017). In H20, it was also shown that the cosmological constraints from KV450 are robust to the specific combination of spectroscopic calibration samples used to obtain the DIR $n(z)$ as long as the spectroscopic datasets provide a sufficient coverage in depth and redshift.

Both DES and HSC calibrate their redshift distributions with a high-quality photometric redshift catalog in the COSMOS field (Laigle et al. 2016). A similar calibration of the KV450 data yielded a $0.6 \sigma$ larger value of $S_{8}(\mathrm{H} 20)$. One hypothesis is that outliers in the COSMOS photo- $z$ catalog cause the estimated redshifts to be biased low. Alternatively, there could be a bias in the fiducial KV450 DIR calibration. Here, we construct mock KV450 and DES-Y1 catalogs based on the MICE2 simulation and quantify the extent to which the redshift distributions might be reliably estimated. As the DES-Y1 data are slightly shallower than KiDS, which matches the depth of the public spectroscopic redshift catalogs, we spectroscopically calibrate the DES-Y1 redshift distributions ${ }^{1}$. Using these newly determined $n(z)$, we evaluate the impact on the cosmological constraints, and perform a combined cosmological analysis with KV450.

\section{KV450 and DES-Y1 cosmological constraints with a homogenized analysis}

To meaningfully compare the cosmological constraints from KV450 and DES-Y1, we begin by homogenizing the cosmological priors and treatment of astrophysical systematic uncertainties (Fig. 1). We consider the KV450 and DES-Y1 measurements and covariance in $\mathrm{H} 20$ and T18, respectively ${ }^{2}$. We do not remeasure the respective data vectors and covariance, and use only the angular scales advocated in $\mathrm{H} 20$ and T18. As KV450 and DES-Y1 observations do not overlap on the sky, we treat the two surveys as distinct.

The cosmological constraints on KV450 and DES-Y1 are obtained using the CosmoLSS ${ }^{3}$ likelihood code (Joudaki et al. 2018) in a Markov chain Monte Carlo (MCMC) analysis. This code has been used to benchmark the LSST-DESC

\footnotetext{
1 The HSC-Y1 shear catalogs were not publicly released at the time of this work, and their greater depth also makes a direct spectroscopic calibration infeasible.

2 A unified analysis of earlier cosmic shear datasets is performed in Chang et al. (2019).

3 https://github.com/sjoudaki/CosmoLSS
}

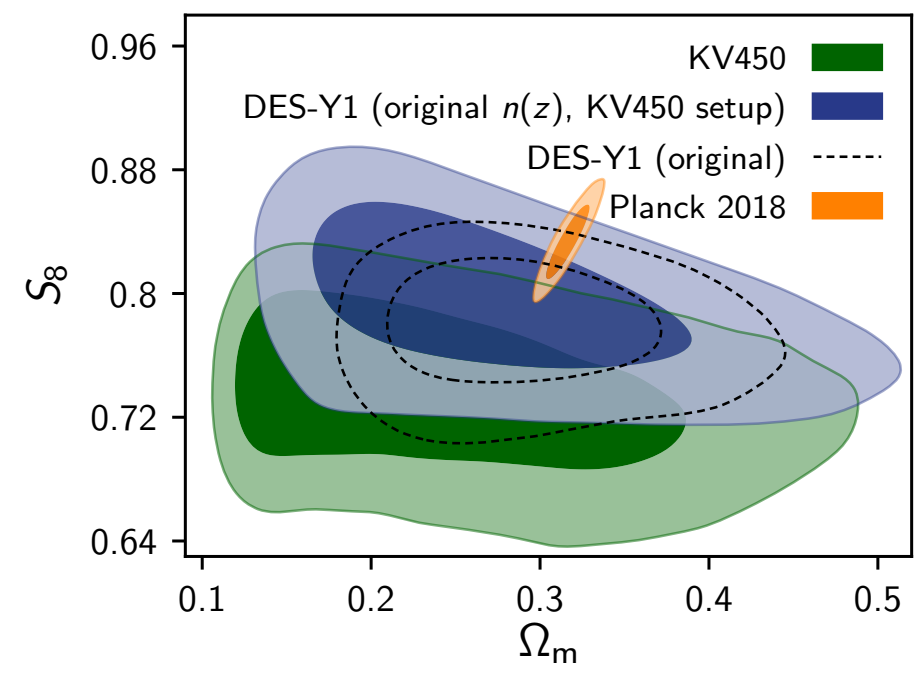

Fig. 1. Marginalized posterior contours in the $S_{8}-\Omega_{\mathrm{m}}$ plane (inner $68 \%$ $\mathrm{CL}$, outer 95\% CL). We show the KV450 constraints in green (solid) using an analysis setup that follows $\mathrm{H} 20$, but including an additional redshift dependence of the IA signal (denoted 'KV450'). In black (dashed), we show the DES-Y1 constraints corresponding to the original T18 analysis, noting that the sum of neutrino masses is varied in this analysis (and hence the contour should not be directly compared with the orange (solid) Planck 2018 contour where the neutrino mass is fixed). The blue (solid) contours show the DES-Y1 constraints where an identical setup to the KV450 analysis is used (along with the original DES-Y1 redshift distributions).

Core Cosmology Library (CCL; Chisari et al. 2019) computation of tomographic cosmic shear, galaxy-galaxy lensing, and galaxy clustering observables. For completeness, we reproduced the COSMOLSS DES-Y1 constraints with both CosMOSIS (Zuntz et al. 2015) and the Planck Collaboration's lensing likelihood in CosmoMC (Planck Collaboration VI 2020). In H20, we moreover showed that the KV450 constraints from CosMoLSS, COSMOSIS, and MONTE PYTHON (Audren et al. 2013) are in excellent agreement.

For both surveys, we implement the cosmological priors of $\mathrm{H} 20$ (see Table 3 therein). In the case of DES-Y1, this includes not only a change in the size of the parameter priors, but notably also a change in the size of the parameter space by fixing the sum of neutrino masses to $0.06 \mathrm{eV}$ instead of varying it freely, a change in the uniform sampling of $A_{\mathrm{s}} \rightarrow \ln \left(10^{10} A_{\mathrm{s}}\right)$, and a change from HALOFIT (Takahashi et al. 2012) to HMCODE (Mead et al. 2015) for the modeling of the nonlinear corrections to the matter power spectrum. Compared to the fiducial DES-Y1 and KV450 analyses, we also switch from MULTINEST (Feroz et al. 2009) to MCMC sampling of the parameter space. Following H20, we allow baryonic feedback to modify the nonlinear matter power spectrum. This does not particularly affect the DES-Y1 constraints given the conservative scale cuts in T18. We keep the shear calibration and photometric redshift uncertainties distinct between the two surveys (given by Table 2 in T18 and Table 3 in H20, respectively).

Conservatively, we allow KV450 and DES-Y1 to have independent parameters governing the IA, using both an amplitude and redshift dependence (as a result, in the combined KV450+DES-Y1 analysis there are 4 free IA parameters). We use a pivot redshift of $z_{0}=0.3$, in agreement with past KiDS analyses and direct measurements of the IA (e.g. Mandelbaum et al. 2011; Joachimi et al. 2011). We find that the $S_{8}$ constraints are robust to the specific treatment of the IA, such 


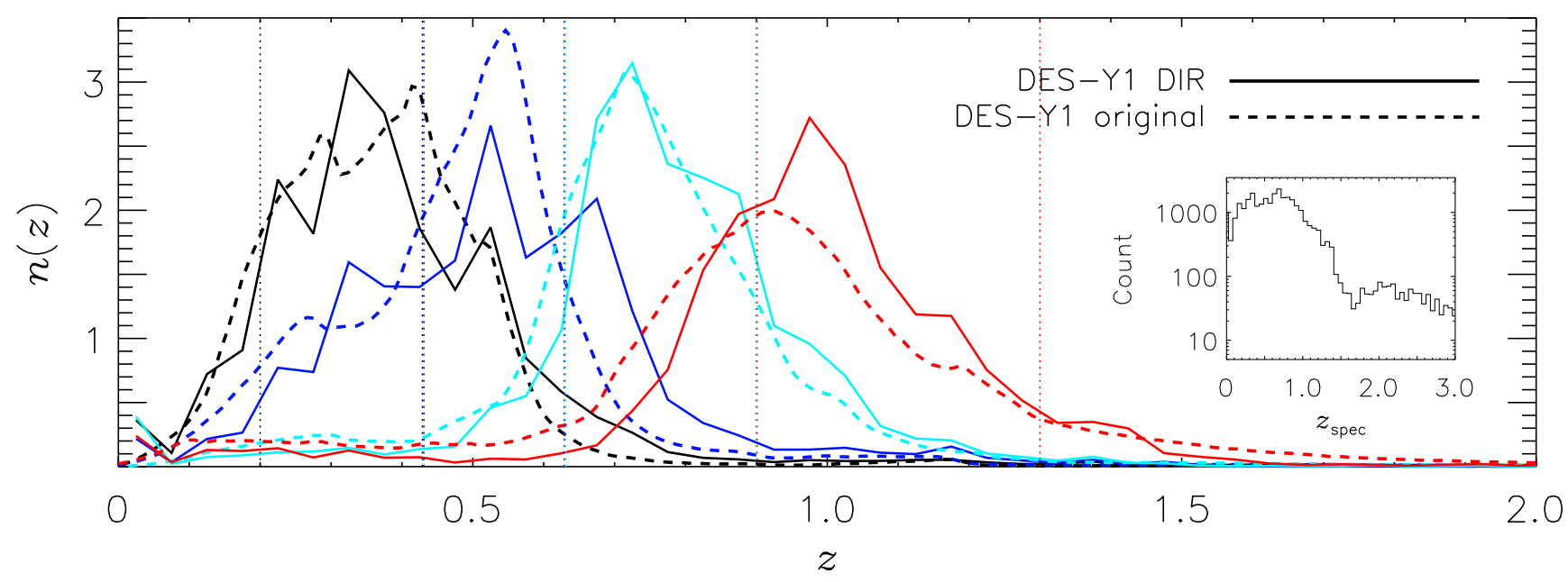

Fig. 2. DES-Y1 redshift distributions for the four tomographic bins (in black, blue, cyan, and red, respectively), showing the publicly released distributions (dashed) and the spectroscopically determined distributions using the DIR approach (solid). The distributions based on spectroscopy are systematically shifted to higher redshifts than the original distributions (accounting for $\Delta z_{i}$ ), and hence favor a lower value of $S_{8}$ than the original DES-Y1 analysis in T18. See Table 1 for the mean redshifts of the different tomographic bins for the two approaches. The vertical dotted lines denote the tomographic bin boundaries. The small inset shows the redshift distribution of the matched photometry/spectroscopy catalog for DES-Y1 containing approximately 30000 objects used in the DIR method. The spectroscopic calibration samples are obtained from zCOSMOS, VVDS-Deep (2h), CDFS, DEEP2 (2h), and VVDS-Wide (22h). We do not show the uncertainties in the $n(z)$ for visual clarity (instead see Table 1 for uncertainties in the mean redshifts).

as removal of the redshift dependence or by assuming that the IA parameters are shared between the two surveys ${ }^{4}$.

We compare the KV450 and DES-Y1 constraints with the Planck 2018 cosmic microwave background (CMB) temperature and polarization measurements (Planck Collaboration VI 2020) $)^{5}$, where the 'TT,TE,EE+lowE' data combination gives $S_{8}=0.834_{-0.016}^{+0.016}$. We exclude the CMB lensing measurements to isolate the high-redshift CMB temperature and polarization constraint on cosmology from the low-redshift Universe.

The KV450 constraint on $S_{8}=0.735_{-0.034}^{+0.042}$ corresponds to a $2.4 \sigma$ discrepancy with Planck 2018. The original DES-Y1 cosmic shear constraint from the publicly released chain ${ }^{6}$ is $S_{8}=0.778_{-0.023}^{+0.030}$ (we note that T18 quotes the marginal posterior maximum of 0.782 instead of the more common posterior mean given here). Compared with the corresponding Planck 2018 result, where the neutrino mass varies, this is a $1.7 \sigma$ difference. The DES-Y1 constraint using the KV450 setup is $S_{8}=0.794_{-0.034}^{+0.037}$, which differs by $1.0 \sigma$ from the Planck 2018 constraint and by $1.1 \sigma$ from the KV450 constraint. This change reflects a shift in the posterior mean and an increase in uncertainty as a result of using HMCODE instead of HALOFIT, wider priors on the amplitude and spectral index of the primordial power spectrum, uniformly sampling $\ln \left(10^{10} A_{\mathrm{s}}\right)$ instead of $A_{\mathrm{s}}$, and fixing the sum of neutrino masses instead of varying it.

We note that when KV450 and DES-Y1 are homogenized to the same assumptions and using the fiducial angular scales, the constraining power of the two datasets is comparable, with the DES-Y1 uncertainty in $S_{8}$ smaller by $8 \%$ (instead of $30 \%$ smaller uncertainty when simply comparing the DES-Y1 con-

\footnotetext{
4 We note, however, that a widened prior on $\eta_{\mathrm{IA}}$ allows for an extended confidence interval at low $\left\{\Omega_{\mathrm{m}}, S_{8}\right\}$ for DES-Y1 alone.

5 Our comparisons are against the public chains as the Planck 2018 likelihoods were not publicly released at the time of this work. This is not fully self-consistent given the mostly narrower prior ranges used by Planck (compared to our KV450 and DES-Y1 runs), but has a negligible impact given the constraining power of the Planck dataset.

6 http://desdr-server.ncsa.illinois.edu/despublic/ y1a1_files/chains/s_13.txt
}

straint in T18 with the KV450 constraint in H20). However, this does not account for the improvement in the DES-Y1 constraining power when extending the scale cuts from the fiducial approach in T18 to better agree with the range of angular scales $\theta$ probed by KV450. We find that such a modification to the angular scales (such that $\left\{\theta_{+}>3, \theta_{-}>7\right\}$ arcmin for all tomographic bin combinations) in our correlation function analysis improves the DES-Y1 uncertainty in $S_{8}$ by approximately $30 \%$ (with a $0.5 \sigma$ decrease in the posterior mean) after marginalizing over baryonic feedback, increasing the deviation from Planck (see also Asgari et al. 2020 for a small-scale analysis with COSEBIs).

\section{Spectroscopic determination of the DES-Y1 source redshift distributions}

The redshift distributions for DES and HSC have so far been obtained by using data from the 30-band photometric dataset 'COSMOS-2015' (Laigle et al. 2016). In HSC-Y1, the fiducial redshift distributions are obtained as a histogram of reweighted COSMOS-2015 photometric redshifts (using the weights of the HSC source galaxies and a self-organizing map, or 'SOM'), and the uncertainties in these distributions are obtained by comparing against the photometric redshift distributions from six different codes where the probability distribution functions of the source galaxy redshifts are stacked (Hikage et al. 2019). In DES-Y1, the Bayesian photometric redshift code BPZ (Benítez 2000) is used to compute a stacked redshift distribution, which is shifted along the redshift axis to best fit a combination of resampled COSMOS-2015 redshift distributions and (for the first three tomographic bins) the clustering of the DES source galaxies and a high-quality photo- $z$ reference sample (REDMAGiC; Rozo et al. 2016) over a limited redshift range (Hoyle et al. 2018).

To compare these approaches to direct spectroscopic determination, which fully decouples the photo- $z$ from the determination of the $n(z), \mathrm{H} 20$ considered a DIR estimate of the KV450 
Table 1. DES-Y1 mean redshifts of the four tomographic bins calibrated with COSMOS-2015 (T18) and spectroscopic redshifts (this work).

\begin{tabular}{lcc}
\hline \hline $\begin{array}{l}\text { Tom. } \\
\text { bin }\end{array}$ & $\begin{array}{c}\text { COSMOS-2015 } \\
\langle z\rangle\end{array}$ & $\begin{array}{c}\text { Spec- } z \text { (DIR) } \\
\langle z\rangle\end{array}$ \\
\hline 1 & $0.389 \pm 0.016$ & $0.403 \pm 0.008$ \\
2 & $0.507 \pm 0.013$ & $0.560 \pm 0.014$ \\
3 & $0.753 \pm 0.011$ & $0.773 \pm 0.011$ \\
4 & $0.949 \pm 0.022$ & $0.984 \pm 0.009$ \\
\hline
\end{tabular}

Notes. The spectroscopic calibration consistently favors distributions with higher mean redshifts compared to COSMOS-2015 (the same is found for the median redshifts). We note that our mock analysis of the spectroscopic calibration based on the MICE2 simulation suggests lower mean redshifts by approximately 0.01-0.03 depending on the tomographic bin (see Appendix A for details on the mock analysis along with a discussion of its limitations and cosmological implications).

redshifts with the help of COSMOS-2015, finding a coherent downward shift in the redshift distributions and a consequent increase in the posterior mean for $S_{8}$. H20 argue that estimating the redshift distributions from COSMOS-2015 might however be unreliable given the 'catastrophic outlier' fraction of $\sim 6 \%$ in the magnitude range $23<i<24$ reported in Laigle et al. $(2016)^{7}$ and a residual photo- $z$ bias of $\left\langle z_{\text {spec }}-z_{\text {phot }}\right\rangle \approx 0.01$ after rejection of outliers. This can be compared to $\sim 1 \%$ unreliable redshifts for the combined spectroscopic calibration sample ${ }^{8}$. The outliers in the COSMOS-2015 photo- $z$ are potentially also more problematic because their effect is most probably asymmetric. Outliers that are truly objects at high- $z$ but are assigned a low COSMOS2015 photo- $z$ are more likely to fall inside the DES-Y1 tomographic bins than outliers that are bona fide low- $z$ galaxies but are assigned a high COSMOS-2015 photo- $z$. Additionally, the bias in the core of the $z_{\mathrm{spec}}-z_{\text {phot }}$ distribution is in the same direction, that is, overall the redshifts might be underestimated by the COSMOS-2015 photo- $z$.

In the DES-Y1 analyses, the case is made that a spectroscopic determination of the source redshift distributions would not be sufficiently accurate due to the incompleteness of the existing spectroscopic surveys at the faint end of the DES observations (Hoyle et al. 2018). We find, however, that even the deeper KV450 source sample is well covered by our spectroscopic compilation, implying that the coverage should also be sufficient for the calibration of the DES-Y1 sample. This is confirmed by a SOM approach to redshift calibration (Masters et al. 2015) presented in Wright et al. (2020).

DES-Y1 overlaps with almost the same deep spectroscopic redshift surveys that were used by H20. As shown in Fig. 2 (inset), this overlap contains some 30000 objects with spectroscopic redshifts from zCOSMOS (Lilly et al. 2009), the DEEP2 Redshift Survey (Newman et al. 2013), the VIMOS VLT Deep Survey (VVDS; Le Fèvre et al. 2013), and the Chandra Deep Field South (CDFS; Vanzella et al. 2008; Popesso et al. 2009; Balestra et al. 2010; Le Fèvre et al. 2013). We find that the $\mathrm{KV} 450$ source sample is well covered as long as spectroscopic redshifts from DEEP2 - the highest-redshift calibration survey are included and the same is true for DES-Y1. However, we note that Hoyle et al. (2018) and Hartley et al. (2020) have moreover

\footnotetext{
7 For $22<i<23$, the outlier rate is significant at 3.5\% (O. Ilbert, priv. comm.).

8 In Wright et al. (2020), we show that the change in the estimated redshift distributions from catastrophic spec- $z$ failures in the spectroscopic compilation is negligible.
}

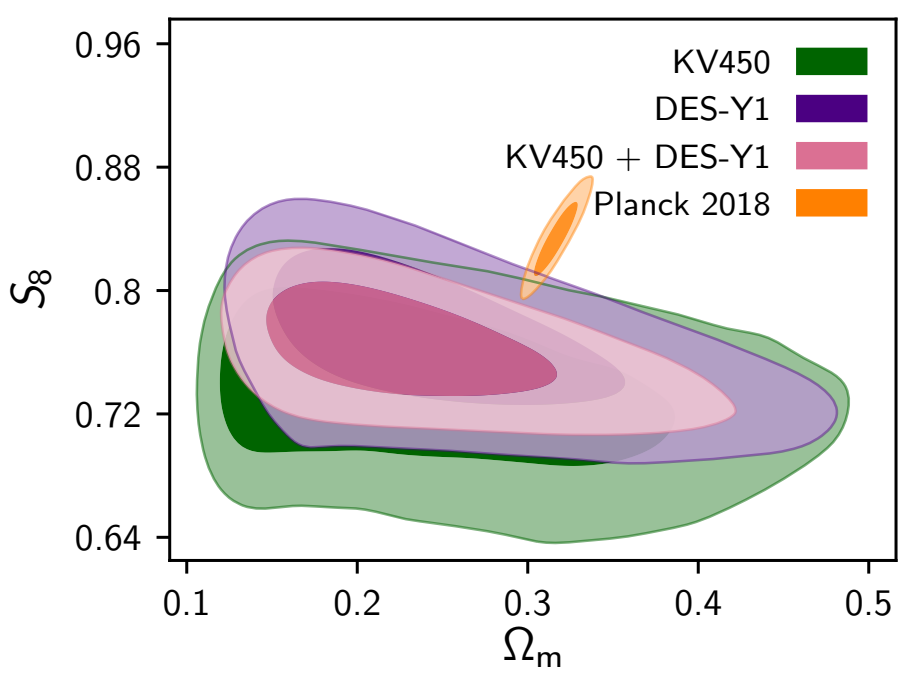

Fig. 3. Marginalized posterior contours in the $S_{8}-\Omega_{\mathrm{m}}$ plane (inner $68 \%$ CL, outer 95\% CL) for KV450 (green), DES-Y1 following a spectroscopic calibration of the redshift distributions and identical setup to the KV450 analysis (purple), the above combined (pink), and Planck 2018 (orange).

argued that the 4-band DES data may be inherently less suitable to our reweighting scheme than the 9-band KiDS+VIKING data, which is a hypothesis that we assess in Appendix A (see also Buchs et al. 2019 for a way to solve this by leveraging the DES Deep fields).

The KV450 and DES-Y1 spectroscopic calibration samples used here differ in detail: DES-Y1 overlaps on the sky with VVDS in both the Deep (2h) and Wide (22h) fields compared to only the Deep ( $2 \mathrm{~h}$ ) field for KV450, and the DES-Y1 calibration does not include the $23 \mathrm{~h}$ field of DEEP2 and the GAMAG15Deep sample (Kafle et al. 2018) which are included in the KV450 calibration. Overall, we obtain the DES-Y1 and KV450 redshift distributions using five and six spectroscopic calibration samples, respectively, four of which are identical ${ }^{9}$. Note that no shear data from these calibration fields are used in both the KiDS and DES cosmological analyses, maintaining independence in the measured shear correlation functions from the two surveys.

Figure 2 shows that the spectroscopic calibration shifts DESY1 redshift distributions to higher redshifts compared to the original photo- $z$ recalibration with COSMOS-2015, consistent with the findings of H20. Mean redshifts of the four tomographic bins are reported in Table 1 for both cases. The spectroscopically determined distributions peak closer to the center of the corresponding tomographic bins, and contain higher-redshift galaxies. These shifts between the spectroscopically estimated and published DES-Y1 $n(z)$ are significant because of their coherence, that is, all tomographic bins shift in the same direction. We emphasize that widening the priors on the uncorrelated $\Delta z_{i}$ nuisance parameters cannot account for such a coherent shift as this is fully degenerate with the cosmological parameters of interest (see the discussion at the end of Sect. 3 in H20).

In Appendix A, we further explore the robustness of the DIR calibration on mock KV450 and DES-Y1 catalogs. This analysis motivates the inclusion of a systematic error model in our analysis to account for potential biases in the DIR calibration. If the error model from the mock survey analysis is fully accurate and representative (see the caveats and discussion in Appendix A),

\footnotetext{
9 Note that the exact area in each of these fields differs slightly between surveys because of the different footprints of KiDS and DES.
} 
the true mean redshifts of the DES-Y1 tomographic bins can be lowered by approximately $0.01-0.03$ compared to the DIR results presented in Fig. 2 and Table 1.

\section{Cosmological impact of DES-Y1 $n(z)$ recalibration and combined constraints with KV450}

We now quantify the impact of the spectroscopic calibration of the DES-Y1 redshift distributions on the cosmological parameter constraints. As it is now on an equal footing with KV450, we moreover perform a combined analysis of the two surveys, shown in Fig. 3.

The DES-Y1 constraint following the spectroscopic calibration of the redshift distributions is $S_{8}=0.765_{-0.031}^{+0.036}$. Compared to using the original redshift distributions, this is a change in the posterior mean by $\Delta S_{8}=-0.029$ and a marginal (5\%) improvement in the $S_{8}$ uncertainty. We verified that this shift in $S_{8}$ is largely recovered by coherently shifting the original DES-Y1 redshift distributions by the $\Delta z_{i}$ difference with the spectroscopically calibrated distributions as reported in Table 1 (i.e. changes in the structure of the $n_{i}(z)$ have a subdominant impact on $S_{8}$ ). This substantial change in the DES-Y1 constraint highlights the importance of the redshift calibration. The size of $\Delta S_{8}$ corresponds to a $0.8 \sigma$ shift in terms of the larger DES uncertainty in the KV450 setup, and a $1.1 \sigma$ shift in terms of the original DESY1 uncertainty quoted in T18. The DES-Y1 constraint using a KV450 analysis setup and spectroscopically calibrated redshift distributions is different from the Planck 2018 constraint on $S_{8}$ by $1.9 \sigma$. The goodness of fit with the spectroscopically calibrated distributions is comparable to that of using the COSMOS2015 distributions (difference in the reduced $\chi^{2}$ by $6 \times 10^{-3}$ ).

Following the homogenization of the analysis setups, the combined KV450+DES-Y1 constraint is $S_{8}=0.762_{-0.024}^{+0.025}$. This is almost exactly a factor of $\sqrt{2}$ improvement in precision compared to KV450 and DES-Y1 on their own. We find a best-fit $\chi^{2}=413.4$ for 397 degrees of freedom, which corresponds to a reduced $\chi^{2}$ of 1.04 and a $p$-value of 0.27 . Using the $\log \mathcal{I}$ statistic (Joudaki et al. 2017) and Jeffreys' scale (Jeffreys 1961; Kass \& Raftery 1995), we find that KV450 and DESY1 are in 'strong' concordance $(\log \mathcal{I}=1.4)$, which is an expected outcome given the $S_{8}$ agreement between the two surveys. The KV450+DES-Y1 constraint is $2.5 \sigma$ discordant with Planck 2018 (we do not evaluate the $\log \mathcal{I}$ statistic in this case as the Planck 2018 likelihood is not public). We note that for the cosmological priors used in T18, the combined KV450+DESY1 dataset is even more discordant with Planck. For this case (not shown in Fig. 3), $S_{8}=0.750_{-0.025}^{+0.022}$, which is a $3.0 \sigma$ discordance with Planck 2018.

The constraints on the astrophysical degrees of freedom, such as the IA amplitude and redshift dependence, do not change significantly in the combined analysis from either survey independently. This is partly a consequence of our analysis decision to keep the KV450 and DES-Y1 intrinsic alignment parameters distinct. We further note that the impact of the spectroscopic calibration for DES-Y1 decreases to $\Delta S_{8}=-0.017$ (from the fiducial $\Delta S_{8}=-0.029$ ) if a systematic error model for the DIR calibration from our study of mock DES data in Appendix A is included in the analysis. In the appendix, we show that a self-consistent change in the redshift distributions of both DES$\mathrm{Y} 1$ and KV450, based on our mocks constructed for each survey, results in effectively the same combined KV450+DES-Y1 constraint on $S_{8}$ as in the fiducial analysis (less than $0.1 \sigma$ difference). While the inclusion of the DEEP2 sample is critical for the redshift calibration of both KV450 and DES-Y1 (Wright et al. 2020), the $S_{8}$ constraints from both surveys are robust to a change in the spec- $z$ calibrating fields to the four fields that they have in common. We note that the spectroscopically calibrated source redshift distributions will have a comparable impact on the $S_{8}$ constraint from the DES-Y1 combined analysis of cosmic shear, galaxy-galaxy lensing, and galaxy clustering (Abbott et al. 2018b).

\section{Conclusions}

We have performed the first combined analysis of Stage-III cosmic shear surveys with KiDS+VIKING-450 and DES-Y1. In obtaining reliable cosmological results, we homogenized the analysis setups and spectroscopically calibrated the DES-Y1 source redshift distributions, both of which have a substantial impact on the parameter constraints. We show that the cosmological constraints from KV450 and DES-Y1 are comparable when analyzed self-consistently over the angular scales advocated by each survey, and that the DES-Y1 constraint on $S_{8}$ changes downward by $0.8 \sigma$ when calibrating the redshift distributions using overlapping deep-field spectroscopy. The combined KV450+DES-Y1 constraint on $S_{8}=0.762_{-0.024}^{+0.025}$ reflects a factor of $\sqrt{2}$ improvement in precision compared to each survey independently, and is $2.5 \sigma$ discordant with the Planck CMB temperature and polarization. This increases to $3.0 \sigma$ when employing the cosmological priors advocated by DES-Y1, and would only increase further by including smaller-scale DES-Y1 measurements sensitive to baryonic feedback.

The substantial change in the DES-Y1 redshift distributions and the corresponding impact on the $S_{8}$ constraint suggests that a similar exercise with HSC-Y1 data would be valuable, and that a self-consistent combined analysis of all three current cosmic shear surveys may sharpen the tension with Planck 2018 even further. We note that the greater depth of HSC (but also future surveys such as the Legacy Survey of Space and Time, LSST) complicates a direct spectroscopic calibration of the redshift distributions and may instead require other approaches such as the cross-correlation between photometric and spectroscopic galaxies (Newman 2008). Ultimately, the advent of additional data expected for KiDS, DES, and HSC in the coming years along with self-consistent combined analyses of cosmic shear surveys will be crucial for resolving the current tension found with the Planck CMB.

Acknowledgements. We thank Chris Blake, Pedro Ferreira, Christos Georgiou, Ian Harrison, Olivier Ilbert, Harry Johnston, Nicolas Martinet, Alexander Mead, Chris Morrison, and Mohammadjavad Vakili for useful discussions. We also thank Ian Harrison for help navigating the public DES data. We thank the DES team and in particular Daniel Grün and Michael Troxel for in-depth discussions that led to the inclusion of the simulation results reported in the appendix. We acknowledge the use of CAMB/COSMOMC packages (Lewis et al. 2000; Lewis \& Bridle 2002). Author contributions: All authors contributed to the development and writing of this Letter. The authorship list is given in three groups: the lead authors (SJ, HHi, DT), followed by two alphabetical groups. The first alphabetical group includes those who are key contributors to both the scientific analysis and the data products. The second group covers those who have either made a significant contribution to the data products or to the scientific analysis. Part of this work was performed using the DiRAC Data Intensive service at Leicester operated by the University of Leicester IT Services, and DiRAC@Durham managed by the Institute for Computational Cosmology, which form part of the STFC DiRAC HPC Facility (https://dirac.ac.uk) acknowledging BEIS and STFC grants STK0003731, STR0023631, STR0010141, STP0022931, STR0023711, STR0008321. We acknowledge support from the European Research Council under grant numbers 693024 (SJ, DT), 770935 (HHi, AHW), $647112(\mathrm{CH}, \mathrm{MA}, \mathrm{TT})$. SJ and DT acknowledge support from the Beecroft 
Trust and STFC. HHi is supported by Emmy Noether (Hi 1495/2-1) and Heisenberg grants (Hi 1495/5-1) of the Deutsche Forschungsgemeinschaft. NEC is supported by a Royal Astronomical Society research fellowship. HHo and AK acknowledge support from Vici grant 639.043.512, financed by the Netherlands Organisation for Scientific Research (NWO). KK acknowledges support by the Alexander von Humboldt Foundation. LM acknowledges support from STFC grant ST/N000919/1. TT acknowledges funding from the European Union's Horizon 2020 research and innovation program under the Marie Sklodowska-Curie grant agreement No 797794. We are indebted to the staff at ESO-Garching and ESO-Paranal for managing the observations at VST and VISTA that yielded the data presented here. Based on observations made with ESO Telescopes at the La Silla Paranal Observatory under programme IDs 177.A-3016, 177.A-3017, 177.A-3018, 179.A- 2004, 298.A-5015, and on data products produced by the KiDS consortium. This project used public archival data from the Dark Energy Survey (DES). Funding for the DES Projects has been provided by the US Department of Energy, the US National Science Foundation, the Ministry of Science and Education of Spain, the Science and Technology Facilities Council of the United Kingdom, the Higher Education Funding Council for England, the National Center for Supercomputing Applications at the University of Illinois at Urbana-Champaign, the Kavli Institute of Cosmological Physics at the University of Chicago, the Center for Cosmology and Astro-Particle Physics at the Ohio State University, the Mitchell Institute for Fundamental Physics and Astronomy at Texas A\&M University, Financiadora de Estudos e Projetos, Fundação Carlos Chagas Filho de Amparo à Pesquisa do Estado do Rio de Janeiro, Conselho Nacional de Desenvolvimento Científico e Tecnológico and the Ministério da Ciência, Tecnologia e Inovação, the Deutsche Forschungsgemeinschaft, and the Collaborating Institutions in the Dark Energy Survey. The Collaborating Institutions are Argonne National Laboratory, the University of California at Santa Cruz, the University of Cambridge, Centro de Investigaciones Energéticas, Medioambientales y Tecnológicas-Madrid, the University of Chicago, University College London, the DES-Brazil Consortium, the University of Edinburgh, the Eidgenössische Technische Hochschule (ETH) Zürich, Fermi National Accelerator Laboratory, the University of Illinois at Urbana-Champaign, the Institut de Ciències de l'Espai (IEEC/CSIC), the Institut de Física d'Altes Energies, Lawrence Berkeley National Laboratory, the Ludwig-Maximilians Universität München and the associated Excellence Cluster Universe, the University of Michigan, the National Optical Astronomy Observatory, the University of Nottingham, The Ohio State University, the OzDES Membership Consortium, the University of Pennsylvania, the University of Portsmouth, SLAC National Accelerator Laboratory, Stanford University, the University of Sussex, and Texas A\&M University. Based in part on observations at Cerro Tololo Inter-American Observatory, National Optical Astronomy Observatory, which is operated by the Association of Universities for Research in Astronomy (AURA) under a cooperative agreement with the National Science Foundation.

\section{References}

Abbott, T. M. C., Abdalla, F. B., Allam, S., et al. 2018a, ApJS, 239, 18 Abbott, T. M. C., Abdalla, F. B., Alarcon, A., et al. 2018b, Phys. Rev. D, 98, 043526

Aihara, H., Armstrong, R., Bickerton, S., et al. 2018, PASJ, 70, S8 Asgari, M., Tröster, T., Heymans, C., et al. 2020, A\&A, 634, A127
Audren, B., Lesgourgues, J., Benabed, K., et al. 2013, JCAP, 2013, 001 Balestra, I., Mainieri, V., Popesso, P., et al. 2010, A\&A, 512, A12 Benítez, N. 2000, ApJ, 536, 571

Buchs, R., Davis, C., Gruen, D., et al. 2019, MNRAS, 489, 820

Chang, C., Wang, M., Dodelson, S., et al. 2019, MNRAS, 482, 3696

Chisari, N. E., Alonso, D., Krause, E., et al. 2019, ApJS, 242, 2

Crocce, M., Castander, F. J., Gaztañaga, E., et al. 2015, MNRAS, 453, 1513

Drlica-Wagner, A., Sevilla-Noarbe, I., Rykoff, E. S., et al. 2018, ApJS, 235 , 33

Edge, A., Sutherland, W., Kuijken, K., et al. 2013, The Messenger, 154, 32

Feroz, F., Hobson, M. P., \& Bridges, M. 2009, MNRAS, 398, 1601

Fosalba, P., Crocce, M., Gaztañaga, E., et al. 2015, MNRAS, 448, 2987

Gruen, D., \& Brimioulle, F. 2017, MNRAS, 468, 769

Hartley, W. G., Chang, C., Samani, S., et al. 2020, MNRAS, submitted [arXiv:2003.10454]

Hikage, C., Oguri, M., Hamana, T., et al. 2019, ApJS, 71, 43

Hildebrandt, H., Viola, M., Heymans, C., et al. 2017, MNRAS, 465, 1454

Hildebrandt, H., Köhlinger, F., van den Busch, J. L., et al. 2020, A\&A, 633, A69

Hoyle, B., Gruen, D., Bernstein, G. M., et al. 2018, MNRAS, 478, 592

Jee, M. J., Tyson, J. A., Hilbert, S., et al. 2016, ApJ, 824, 77

Jeffreys, H. 1961, Theory of Probability, 3rd edn. (Oxford, UK: OUP)

Joachimi, B., Mandelbaum, R., Abdalla, F. B., et al. 2011, A\&A, 527, A26

Johnson, A., Blake, C., Amon, A., et al. 2017, MNRAS, 465, 4118

Joudaki, S., Blake, C., Heymans, C., et al. 2017, MNRAS, 465, 2033

Joudaki, S., Blake, C., Johnson, A., et al. 2018, MNRAS, 474, 4894

Kafle, P. R., Robotham, A. S. G., Driver, S. P., et al. 2018, MNRAS, 479, 3746

Kass, R. E., \& Raftery, A. E. 1995, J. Am. Stat. Assoc., 90, 773

Kuijken, K., Heymans, C., Hildebrandt, H., et al. 2015, MNRAS, 454, 3500

Laigle, C., McCracken, H. J., Ilbert, O., et al. 2016, ApJS, 224, 24

Le Fèvre, O., Vettolani, G., Garilli, B., et al. 2005, A\&A, 439, 845

Le Fèvre, O., Cassata, P., Cucciati, O., et al. 2013, A\&A, 559, A14

Lewis, A., \& Bridle, S. 2002, Phys. Rev. D, 66, 103511

Lewis, A., Challinor, A., \& Lasenby, A. 2000, ApJ, 538, 473

Lilly, S. J., Le Fèvre, O., Renzini, A., et al. 2007, ApJS, 172, 70

Lilly, S. J., Le Brun, V., Maier, C., et al. 2009, ApJS, 184, 218

Lima, M., Cunha, C. E., Oyaizu, H., et al. 2008, MNRAS, 390, 118

Mandelbaum, R. 2018, ARA\&A, 56, 393

Mandelbaum, R., Blake, C., Bridle, S., et al. 2011, MNRAS, 410, 844

Masters, D., Capak, P., Stern, D., et al. 2015, ApJ, 813, 53

Mead, A. J., Peacock, J. A., Heymans, C., et al. 2015, MNRAS, 454, 1958

Morrison, C. B., Hildebrandt, H., Schmidt, S. J., et al. 2017, MNRAS, 467, 3576

Newman, J. A. 2008, ApJ, 684, 88

Newman, J. A., Cooper, M. C., Davis, M., et al. 2013, ApJS, 208, 5

Planck Collaboration VI. 2020, A\&A, in press, https://doi.org/10.1051/ 0004-6361/201833910

Popesso, P., Dickinson, M., Nonino, M., et al. 2009, A\&A, 494, 443

Rozo, E., Rykoff, E. S., Abate, A., et al. 2016, MNRAS, 461, 1431

Takahashi, R., Sato, M., Nishimichi, T., et al. 2012, ApJ, 761, 152

Troxel, M. A., MacCrann, N., Zuntz, J., et al. 2018, Phys. Rev. D, 98, 043528

Vanzella, E., Cristiani, S., Dickinson, M., et al. 2008, A\&A, 478, 83

Wright, A. H., Hildebrandt, H., Kuijken, K., et al. 2019, A\&A, 632, A34

Wright, A. H., Hildebrandt, H., van den Busch, J. L., et al. 2020, A\&A, 637, A100

Zuntz, J., Paterno, M., Jennings, E., et al. 2015, Astron. Comput., 12, 45 


\section{Appendix A: Tests on MICE2 mock catalogs}

We test the spectroscopic DIR calibration described in Sect. 3 on mock catalogs created from the public MICE2 simulation (Fosalba et al. 2015; Crocce et al. 2015). These mock catalogs are similar to the ones used in Wright et al. (2020) and will be described in detail in van den Busch et al. (in prep.) for KV450. Here, we further describe how the mock catalogs are designed to resemble the DES-Y1 data. It is important to stress that this exercise is not meant to produce fully realistic mock catalogs that resemble the data in all aspects. Rather, it is aimed at producing mock catalogs that are similarly complex as the data. As such, the mock catalogs can be used to inform us about the expected size of systematic uncertainties in the DIR calibration.

We first estimate the observed size and shape of each simulated galaxy by taking the semi major and minor axes reported in the MICE2 catalog and adding the seeing (from Drlica-Wagner et al. 2018) in quadrature. Together with the $10 \sigma$ limiting magnitudes quoted in Drlica-Wagner et al. (2018) we estimate the noise level of the evolution-corrected model magnitudes. Subsequently, drawing from the corresponding Gaussian distributions, we create a noise realization for each galaxy in each band and recalculate the magnitude uncertainty based on this realization. This yields a catalog of 'observed' magnitudes and their errors. We found that treating the limiting magnitudes from Drlica-Wagner et al. (2018) as $10 \sigma$ limits in this way results in a mock catalog that is too shallow compared to the data, which might be attributed to aperture effects. Deliberately adapting the limits to $12 \sigma$ yields a good match between data and mocks in terms of the noise level in the four DES bands. We note that we include weak-lensing magnification in all magnitude estimates although this particular aspect has virtually no impact on the results.

Subsequently, we match each mock galaxy to its nearest neighbor in the data catalog in 4-dimensional magnitude space and assign it the responsivity weight of that galaxy in the data. This yields a properly weighted mock source sample. We run BPZ to estimate photo- $z$ for the mock galaxies using the setup described in Hildebrandt et al. (2020), but restricting the redshift range to that of MICE2 $(0.06<z<1.4)^{10}$. This setup differs slightly from what is done in DES-Y1 (Hoyle et al. 2018) but the properties of the resulting photo- $z$ (scatter, bias, and outlier rate as a function of photo- $z$ and magnitude) are very similar to what is seen when comparing the DES 4-band photo- $z$ to the combined spec- $z$ sample on the data.

The next crucial step is to select samples from the mock catalog that resemble the spec- $z$ samples used in the DIR analysis presented in Sect. 3. Here, we apply the same selection criteria as the zCOSMOS ( $i<22.5$; Lilly et al. 2007), VVDS ( $i<24$; Le Fèvre et al. 2005, 2013), and DEEP2 $(R<24.1$ plus color selection; Newman et al. 2013) teams to areas that correspond to the areas sampled by the data. Moreover, we implement the magnitude- and partly also redshift-dependent spectroscopic success rates reported in those papers. Where necessary, we further downsample the catalogs to yield numbers comparable to the data. This is required because the number density as a function of redshift is not identical in the simulation and the real Universe.

We find that the fiducial DEEP2 color selection yields a redshift distribution that looks somewhat different from the one in the data. This is probably due to the fact that galaxy colors in MICE2 are not fully realistic, especially at high redshift. Inspect-

\footnotetext{
${ }^{10}$ We also run a setup with a wider fitting range of $0<z<7$ and do not find any significant changes in the results.
}

Table A.1. KV450 and DES-Y1 changes in the mean redshift for each tomographic bin informed by the MICE2 mock catalogs (i.e. Truth -

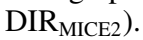

\begin{tabular}{lcc}
\hline \hline $\begin{array}{l}\text { Tom. } \\
\text { bin }\end{array}$ & KV450 & DES-Y1 \\
& $\Delta\langle z\rangle$ & $\Delta\langle z\rangle$ \\
\hline 1 & $-0.048 \pm 0.010$ & $-0.026 \pm 0.016$ \\
2 & $-0.026 \pm 0.008$ & $-0.021 \pm 0.014$ \\
3 & $-0.033 \pm 0.012$ & $-0.033 \pm 0.010$ \\
4 & $0.005 \pm 0.008$ & $-0.012 \pm 0.012$ \\
5 & $0.013 \pm 0.008$ & - \\
\hline
\end{tabular}

Notes. These MICE2 uncertainties have conservatively been multiplied by a factor of two to account for the inherent limitations of the mock catalogs.

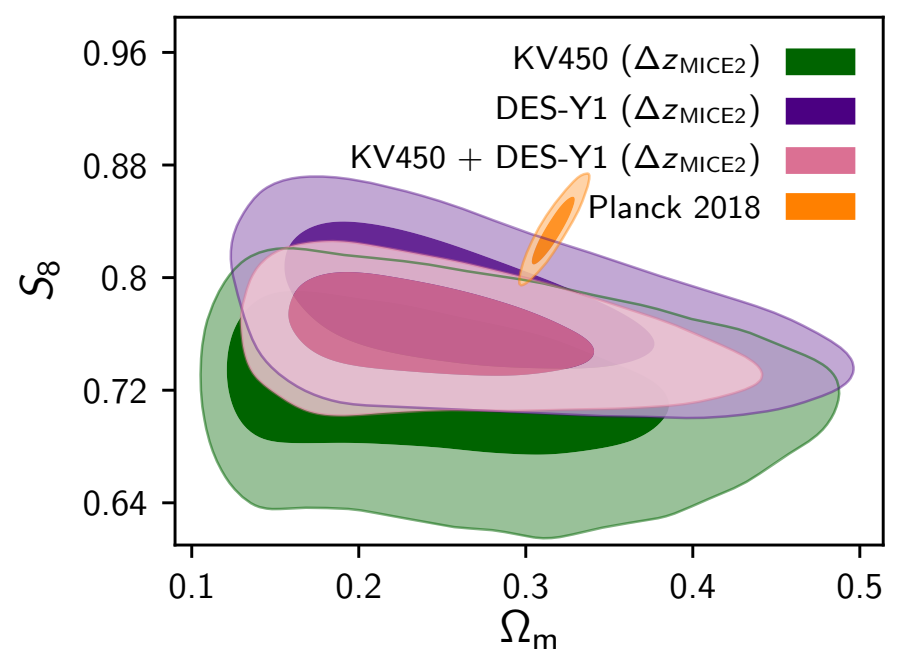

Fig. A.1. Marginalized posterior contours in the $S_{8}-\Omega_{\mathrm{m}}$ plane (inner $68 \% \mathrm{CL}$, outer $95 \% \mathrm{CL}$ ) following an alternative analysis of the cosmic shear datasets with MICE2 priors on the $\Delta z_{i}$ parameters. We show KV450 in green, DES-Y1 in purple, KV450+DES-Y1 in pink, and Planck 2018 in orange.

ing the location of those high- $z$ galaxies that are supposed to be targeted by DEEP2 in $B-R$ versus $R-I$ color space, we slightly adapt those criteria to take the slightly different colors of MICE2 galaxies into account. This yields a better match to the observed spectroscopic redshift distribution. In the end, this adaptation does not have a strong influence on the results as we find by running tests with the original as well as the adapted cuts.

We select tomographic bins from the MICE2 realization of the DES-Y1 data and calibrate those with the DIR method using the mock spec- $z$ samples described above. Comparing the true mean redshifts of the galaxies in those four tomographic bins to the ones estimated from DIR on the mocks yields offsets of $\Delta z_{1}=\left\langle z_{1}\right\rangle_{\text {True }}-\left\langle z_{1}\right\rangle_{\text {DIR }}=-0.026, \Delta z_{2}=-0.021, \Delta z_{3}=-0.033$, and $\Delta z_{4}=-0.012$ (see also Table A.1). The exact values depend somewhat on the exact definition of the mock spec- $z$ sample.

These results indicate that we might overestimate the true redshifts of the tomographic bins and hence underestimate $S_{8}$, an effect opposite - albeit smaller - to the one seen when replacing the original DES $n(z)$ with our spectroscopic recalibration. This could be attributed to the color preselection of DEEP2 in combination with a magnitude space that is limited to four dimensions, such that the photometric information from the DES griz filters alone is not capable of accurately breaking color-redshift degeneracies and down-weighting the high- $z$ DEEP2 galaxies. This 
problem was already noted in Gruen \& Brimioulle (2017) using a similar technique. While MICE2 shows an impressive similarity to the real Universe, there is certainly the caveat that the simulation is limited to $z<1.4$. The modeling of high- $z$ tails is therefore not possible. The issue with the colors of high- $z$ galaxies further illustrates the limitations of such a mock. Whether these results hold with a mock catalog extending farther in redshift remains to be seen and will be investigated in future work ${ }^{11}$.

The DES-Y1 biases are comparable in size to what was found on very similar mocks resembling the KV450 data, as reported in Wright et al. (2020) and Table A.1. While the DIR calibration on 9-band KV450 data should be less prone to systematic uncertainties than the one on the 4-band DES-Y1 data, we suppose that the greater depth of KV450 complicates the calibration and leads to biases of the same order. Despite these limitations, the biases found in the mock analysis give an indication of the systematic error inherent in the DIR calibration with typical spectroscopic catalogs. In order to address this concern, we run another cosmology parameter analysis where we apply these shifts by centering the priors on the $\Delta z_{i}$ parameters on these values instead of zero. As an uncertainty we use the standard deviation over 100 lines of sight in the mocks but multiply this by an arbitrary factor of two to account for limitations in the simulation.

Following this approach, we find that $S_{8}=0.721_{-0.032}^{+0.045}$ for $\mathrm{KV} 450, S_{8}=0.777_{-0.033}^{+0.035}$ for DES-Y1, and $S_{8}=0.760_{-0.023}^{+0.026}$ for KV450+DES-Y1 (as shown in Fig. A.1). These constraints correspond to changes of $\Delta S_{8}=[-0.014,+0.012,-0.002]$ and $\Delta \chi^{2}=[0.52,2.0,2.8]^{12}$ relative to our fiducial results in the main body of the paper for KV450, DES-Y1, and KV450+DES-Y1, respectively. Given the comparable size of the applied MICE2 and bootstrap errors on the $\Delta z_{i}$ parameters, we do not find significant differences in the size of the $S_{8}$ uncertainties (the largest difference corresponds to a $2 \%$ increase in the uncertainty).

We note that the KV450 constraint on $S_{8}$ shifts toward lower values despite substantial negative $\Delta z_{i}$ shifts in the first three tomographic bins. This is explained by the greater constraining power of the higher redshift fourth and fifth bins which exhibit positive shifts in their mean redshifts. The change in $S_{8}$ is -0.017 for DES-Y1 relative to the COSMOS-2015 calibrated redshift distributions, which corresponds to a $0.5 \sigma$ shift in terms of the larger DES-Y1 uncertainty in the KV450 setup (as compared to the fiducial shift of $0.8 \sigma$; noting the significance of both shifts increase in terms of the original DES-Y1 uncertainty). In other words, the MICE2 mocks suggest that the redshift distributions from the pre-revised DIR in the case of KV450 and from COSMOS-2015 in the case of DES-Y1 both result in an overestimated posterior mean of $S_{8}$ (by 0.014 and 0.017 , respectively). Here, the concordance in the MICE2-revised $S_{8}$ constraints of KV450 and DES-Y1 is at the $1.1 \sigma$ level (as compared to the stronger fiducial concordance in $S_{8}$ of $0.6 \sigma$ ), while the combined KV450+DES-Y1 constraint on $S_{8}$ remains unchanged at $0.1 \sigma$ relative to the fiducial result.

In summary, this analysis on the MICE2 mocks illustrates the importance of realistic mock catalogs for future analyses of weak-lensing surveys. In particular, a very wide redshift range is desirable to properly account for photo- $z$ outliers in the systematic error estimates.

\footnotetext{
${ }^{11}$ See also Hartley et al. (2020) who conduct a similar simulated analysis with a different calibration sample.

12 These changes in the goodness of fit are obtained for data vectors of $\{195,227,422\}$ elements in size and thereby $\{180,211,397\}$ degrees of freedom for KV450, DES-Y1, and KV450+DES-Y1, respectively.
} 